\title{
Unsteady Validation of a Mean Flow Boundary Condition for Computational Aeroacoustics
}

\author{
R. Hixon and F. Zhen \\ University of Toledo \\ Toledo, OH 43606 \\ M. Nallasamy \\ QSS Group, Inc. \\ NASA Glenn Research Center \\ Cleveland, $\mathrm{OH} 44135$ \\ S. Sawyer \\ Mechanical Engineering Department \\ The University of Akron \\ Akron, $\mathrm{OH} 44325$ \\ R. Dyson \\ NASA Glenn Research Center \\ Cleveland, $\mathrm{OH} 44135$
}

Mechanical, Industrial, and Manufacturing Engineering Department

\begin{abstract}
In this work, a previously developed mean flow boundary condition will be validated for unsteady flows. The test cases will be several reference benchmark flows consisting of vortical gusts convecting in a uniform mean flow, as well as the more realistic case of a vortical gust impinging on a loaded $2 \mathrm{D}$ cascade. The results will verify that the mean flow boundary condition both imposes the desired mean flow as well as having little or no effect on the instantaneous unsteady solution.
\end{abstract}

\section{Introduction}

The field of Computational Aeroacoustics (CAA) is concerned with the time-accurate calculation of unsteady flow fields. The flow problems of interest can be divided into two types: initial-value, and long-time unsteady problems. In an initial-value problem, the initial flow solution is known exactly, and the computation is focused on obtaining a time-accurate unsteady solution throughout the calculation. In a long-time unsteady problem, the initial flow field is not correctly specified, and the solution must evolve over time until it converges to the long-time unsteady flow.

It is relatively rare to solve an initial-value flow problem, mainly because the initial solution must be exactly specified. On the other hand, many realistic flow problems can be classified as long-time unsteady. Unlike an initial-value problem, where the entire calculation must be highly accurate, the long-time unsteady flow calculation can be 
divided into two distinct phases.

In the first phase, an initial flow solution is specified; since this is generally not the correct long-time solution, the flow evolves through a transient phase before converging to a long-time unsteady solution. During the transient phase, the goal is to converge the flow to the long-time unsteady solution rapidly; thus the transient computation need not be highly accurate in time.

Once the flow has converged to the long-time unsteady solution, the second phase of the computation begins. Here, the desired unsteady flow data is gathered; thus, a highly time-accurate calculation is desired. The data gathered in this phase is the desired output for the flow simulation.

The boundary conditions used for each phase of the calculation should be chosen to achieve the goals of each phase. Currently, nonreflective or damping boundary conditions are used for most CAA calculations. ${ }^{1}$ These conditions are designed to allow outgoing disturbances to exit the computational domain without generating either real or spurious incoming disturbances. These conditions are ideal for either an initial-value computation or the data-gathering phase of a long-time unsteady computation, when the flow solution correctly represents the desired mean flow.

However, these nonreflective boundary conditions have no mechanism for maintaining a desired mean flow when implemented in a nonlinear flow solver. In the initial transient phase, large disturbances may propagate through the boundary and exit the domain. These disturbances affect the flow solution at the boundary, and the correct method to 'reset' the flow at the boundary is to impose an incoming disturbance originating outside of the computational domain. In previous work, such a 'mean flow boundary condition', or MFBC, has been demonstrated on steady flows, showing the ability to obtain a desired mean flow regardless of the initial flow conditions. ${ }^{2}$

Notice that in this work, the mean flow boundary condition does not act on the instantaneous flow solution at the boundary; instead, it acts on the time-averaged mean flow at the boundary. In this way, the instantaneous outgoing waves that do not affect the mean flow will not be reflected. The proposed work will investigate the validity of the $\mathrm{MFBC}$ approach for unsteady flows.

\section{Governing Equations and Numerical Method}

In this work, the Euler equations are solved. The 2D nonlinear Euler equations may be written in Cartesian form as:

$$
Q_{t}+E_{x}+F_{y}=0
$$

The NASA Glenn Research Center BASS code was used to solve this equation.-6 The BASS code uses optimized explicit time marching combined with high-accuracy finitedifferences to accurately compute the unsteady flow. The code is parallel, and uses a block-structured curvilinear grid to represent the physical flow domain. A constant- 
coefficient $10^{\text {th }}$ order artificial dissipation $\operatorname{model}^{7}$ is used to remove unresolved highfrequency modes from the computed solution.

The BASS code solves the Euler equations using the nonconservative chain-rule formulation; previous experience has indicated that the formal lack of conservation is offset by the increased accuracy of the transformed equations. ${ }^{8.9}$ The chain-rule form of the Euler equations are:

$$
\begin{gathered}
Q_{t}+\xi_{t} Q_{\xi}+\eta_{t} Q_{\eta} \\
+\xi_{x} E_{\xi}+\eta_{x} E_{\eta} \\
+\xi_{y} F_{\xi}+\eta_{y} F_{\eta}=0
\end{gathered}
$$

For this work, the optimized low-storage RK56 scheme of Stanescu and Habashi ${ }^{10}$ was combined with the prefactored sixth-order compact differencing scheme of Hixon ${ }^{11}$.

\section{Previous Work}

In previous work, the MFBC has been tested for convergence to a desired steady mean flow, though the solution procedure was a fully unsteady nonlinear time-marching method. The previous test case was a 2D loaded cascade, with a flow turning angle of 36 degrees. The desired mean flow conditions were:

$$
\begin{gathered}
\left(\bar{p}_{o}\right)_{\text {inflow }}=1.0 \\
\left(\bar{T}_{o}\right)_{\text {inflow }}=1.0 \\
(\bar{\alpha})_{\text {inflow }}=1.0 \\
(\bar{p})_{\text {oufflow }}=0.92
\end{gathered}
$$

Figure 1 shows the ability of the MFBC to obtain the desired mean flow, while the result without the MFBC obtains an incorrect mean flow. Reference 2 gave a wide variety of initial conditions and showed the ability of the MFBC to converge to the desired mean flow regardless of the initial flow conditions.

\section{Proposed Work}

For this validation effort, two types of test problems will be chosen. The first problems have low-amplitude vortical gusts convecting in a uniform mean flow. The second problem will be the CAA benchmark problem of a vortical gust impinging on a loaded 2D cascade. ${ }^{12}$ The first problems have an analytic solution, allowing very precise validation. The second problem will have a benchmark solution to compare with. As an extension of the previous work, the instantaneous nonreflecting boundary condition of Giles $^{13}$ will be combined with the characteristic-based ${ }^{14,15}$ mean flow boundary condition. The results will show the utility of the new mean flow boundary condition. 


\section{References}

1) Hixon, R. ' Radiation and Wall Boundary Conditions for Computational Aeroacoustics: A Review' International Journal of Computational Fluid Dynamics, 2003.

2) Hixon, R., Nallasamy, M., Sawyer, S., and Dyson, R., ' MeanFlow Boundary Conditions for Computational Aeroacoustics' , AIAA Paper 2003-3299, May 2003.

3) Hixon, R, Nallasamy, M., and Sawyer, S., ' ParallelizationStrategy for an Explicit Computational Aeroacoustics Code' , AIAA Paper 2002-2583, July 2002.

4) Hixon, R., Nallasamy, M., and Sawyer, S., ' Effect of Grid Singularities on the Solution Accuracy of a CAA Code' , AIAA Paper 2003-0879, Jan. 2003.

5) Nallasamy, M., Hixon, R., Sawyer, S., Dyson, R., and Koch, L., ' Parallel Simulation of Rotor Wake - Stator Interaction Noise' , AIAA Paper 2003-3134, May 2003.

6) Sawyer, S., Nallasamy, M., Hixon, R., Dyson, R., and Koch, D., ' Computational Aeroacoustic Prediction of Discrete-Frequency Noise Generated by a Rotor-Stator Interaction' , AIAA Paper 2003-3268, May 2003.

7) Kennedy, C. A. and Carpenter, M. H., ' SeveralNew Numerical Methods for Compressible Shear-Layer Simulations' Applied Numerical Mathematics, Vol. 14, 1994, pp. 397-433.

8) Hixon, R., Shih, S.-H., and Mankbadi, R. R., 'Evaluation of Boundary Conditions for the Gust- Cascade Problem', Journal of Propulsion and Power, Vol. 16, No. 1, 2000, pp. 72-78.

9) Hixon, R., Mankbadi, R. R., and Scott, J. R., ' Validatiomf a High-Order Prefactored Compact Code on Nonlinear Flows with Complex Geometries' AIAA Paper 20011103, Jan. 2001.

10)Stanescu, D. and Habashi, W. G., ' $2 \mathrm{~N}$-StorageLow Dissipation and Dispersion Runge- Kutta Schemes for Computational Acoustics' ,Journal of Computational Physics, Vol. 143, No. 2, 1998, p. 674-681.

11)Hixon, R., ' PrefactoredSmall-Stencil Compact Schemes' Journal of Computational Physics, Vol. 165, 2000, p. 522-541.

12)www.math.fsu.edu/caa4

13)Giles, M. B., ' NonreflectingBoundary Conditions for Euler Equation Calculations' , AIAA Journal, Vol. 28, No. 12, 1990, pp. 2050-2058.

14)Thompson, K. W., ' TimeDependent Boundary Conditions for Hyperbolic Systems' , Journal of Computational Physics, Vol. 68, No. 1, 1987, pp. 1-24.

15) Thompson, K. W., ' Timdependent Boundary Conditions for Hyperbolic Systems II' Journal of Computational Physics, Vol. 89, 1990, pp. 439-461. 


\section{Figures}

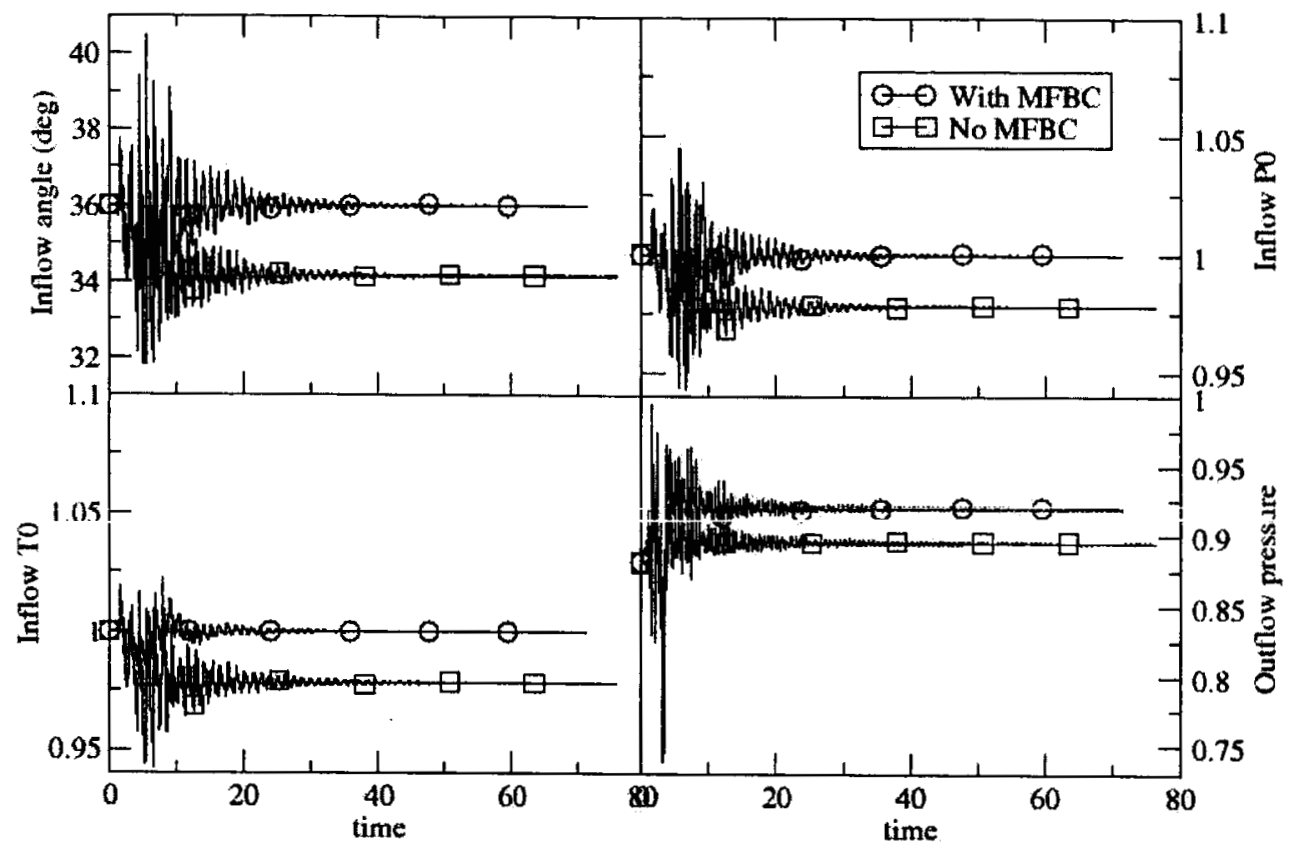

Figure 1: Effect of Mean Flow Boundary Condition on the Flow Obtained by BASS Code on 2D Loaded Cascade Test Case 